\title{
Consideration of the Self-Care Supporting Program Including Simple Exercise for Patients with Early Stages of Secondary Lower-Limb Lymphedema
}

\author{
Chitose Arakawa ${ }^{1,2}$, Chiharu Akazawa ${ }^{3}$, Sayoko Teraguchi ${ }^{3}$ \\ ${ }^{1}$ Graduate School of Nursing, Osaka Medical College, Osaka, Japan \\ ${ }^{2}$ School of Human Nursing, The University of Shiga Prefecture, Shiga, Japan \\ ${ }^{3}$ Faculty of Nursing, Osaka Medical College, Osaka, Japan \\ Email: arakawa.c@nurse.usp.ac.jp,fon137@osaka-med.ac.jp
}

How to cite this paper: Arakawa, C., Akazawa, C. and Teraguchi, S. (2021) Consideration of the Self-Care Supporting Program Including Simple Exercise for Patients with Early Stages of Secondary Lower-Limb Lymphedema. Health, 13, 238-252.

https://doi.org/10.4236/health.2021.133020

Received: January 3, 2021

Accepted: March 12, 2021

Published: March 15, 2021

Copyright $\odot 2021$ by author(s) and Scientific Research Publishing Inc. This work is licensed under the Creative Commons Attribution International License (CC BY 4.0).

http://creativecommons.org/licenses/by/4.0/

\begin{abstract}
Aim: We devised a self-care supporting program targeting patients with early stages of secondary lower-limb lymphedema. The program incorporates "Simple exercises to replace lymphatic drainage" based on lymphatic flow. The purpose of this study was to consider the feasibility of continuing this program. Methods: The participants were patients in the early stages of secondary lower-limb lymphedema after gynecological cancer surgery and lymphedema therapists with more than five years of experience. Patients continued self-care at home after being briefed on the program, and they were analyzed on their self-care continuity status one month later based on a self-administered questionnaire survey and self-care notes. We interviewed the lymphedema therapists about this program to discuss the feasibility of continuing it and obtain feedback. Results: The patients who participated in the study were six women who underwent surgery with lymph node dissection for gynecological cancer. The therapists were five nurses and one occupational therapist. The patient understood the importance of all items in the self-care. "Observation," "Touching," and "Skin care" were relatively easy to continue. "Lymph drainage" and "Exercise" were continued with "Simple exercises to replace lymphatic drainage". "Other exercises" were able to continue by adding distance and time to daily activities. Furthermore, "Measurement" and "Recording" became a burden and were difficult to continue. Nonetheless, this program was generally approved by the lymphedema therapists. They also pointed out the content and format of the self-care notes as improvements. Conclusions: We found that the self-care supporting program that incorpo-
\end{abstract}


rated "Simple exercises to replace lymphatic drainage" might be feasible to continue self-care for patients with early stages of secondary lower-limb lymphedema. Furthermore, we found that we needed to improve the "Measurement" and "Recording" sections of this program.

\section{Keywords}

Secondary Lower-Limb Lymphedema, Early Stages, Self-Care Supporting Program, Lymphatic Drainage, Exercises

\section{Introduction}

"Secondary lower-limb lymphedema" (lymphedema) is often caused by lymph node dissection with surgery or radiation therapy for treating such as endometrial cancer, cervical cancer, ovarian cancer, bladder cancer, prostate cancer. Once lymphedema develops, it does not respond to the treatment and troubles all aspects of life. For example, the patient may find it difficult to continue daily activities due to the pain and limited range of motion of joints, daily work, and put on or take off clothes. According to nationwide surveys conducted in 2003 and 2013 by the "Sociology of Cancer" research group in Japan, the top concerns and burdens among patients and survivors of uterine cancer were "swelling condition due to lymphedema" and "symptoms due to lymphedema" respectively [1]. The incidence of lymphedema after surgery for treating endometrial cancer, cervical cancer, and ovarian cancer (gynecological cancer) is about $20 \%-25 \%$. Since the number of new patients per year is about 40,000 [2], it is estimated that lymphedema occurs in about 10,000 patients every year.

The degree of lymphedema progression is classified by the International Society of Lymphology (ISL) [3]. Stage 0 refers to a latent or subclinical condition where swelling is not yet evident despite the impaired lymph transport, subtle alterations in tissue fluid/composition, and changes in subjective symptoms. Stage I represents an early accumulation of fluid relatively high in protein content, which subsides with limb elevation. Stage II involves changes in solid structures, limb elevation alone rarely reduces tissue swelling, and pitting occurs. Later in Stage II, the limb may not pit as excess subcutaneous fat and fibrosis develop. Stage III encompasses lymphostatic elephantiasis where pitting can be absent and trophic skin changes such as acanthosis, alterations in skin character and thickness, further deposition of fat and fibrosis, and warty overgrowths develop [4]. All patients who have undergone surgery with lymph node dissection are stage 0 and have pre-lymphedema.

The treatment and care of lymphedema involve a surgical treatment (e.g., lymphatic venous anastomosis) and a conservative treatment. As a conservative treatment, Complete Decongestive Therapy (CDT) includes skin care, medical manual lymphatic drainage (lymphatic drainage), compression therapy using elastic bandages and compression garments, exercise with compression, and daily 
life guidance [5] [6]. There is no curative treatment for lymphedema, and it is important to take care of the patient as early as possible after lymphedema develops and prevent their condition from deteriorating.

In Japan, a "lymphedema guidance and management fee" has been newly set up as a medical fee since 2008 [7], and the number of medical institutions that provide lymphedema prevention guidance before discharge or lymphedema outpatient clinics has increased. In addition, subsequent revisions have expanded the scope of reimbursement by increasing the frequency of guidance. However, the reimbursement scope is mainly conducted for patients in a severe stage (stage II or later). In addition, during the period of before and after hospital discharge, when lymphedema prevention guidance is provided, patients are more likely interested in their medical condition and future treatment and are less concerned about lymphedema, which may or may not occur, keeping them engaged in self-care program for prevention. Therefore, patients come to the outpatient clinic for treatment of lymphedema later in Stage II or when they are suffering from severe physical pain and face obstacles in daily life. During this period, the edema has become irreversible, resulting in the deterioration of their physical functions, leading to a decrease in Quality of Life (QOL).

As a previous study on self-care for lymphedema, based on a questionnaire survey, the Akazawa team found that about $20 \%$ of the patients were not able to continue self-care, and those who failed to do that quit in less than a month [8]. The reasons that were reported are "time-consuming," "troublesome," and "cannot feel the effect." In response to the "time-consuming" and "troublesome," the Akazawa team used "Bioelectrical Impedance Analysis"(BIA) to evaluate the distribution and movement of water in the body by lymphatic drainage of the upper body only and suggested that drainage of the upper body should get a good result in stage 0 to stage I [9]. In response to the "cannot feel the effect" reason, the authors reported that self-efficacy for continuation tends to be maintained by quantifying the edema status using a simple body composition meter and "visualizing" the status through recording [10].

As a previous study on the effects of exercise on lymphedema, Lymphedema Clinical Practice Guideline in Japan states that for secondary upper-limb lymphedema, "exercise therapy with load reduces the incidence of lymphedema and improves muscle strength (A2: strongly recommended)" [11]. However, for secondary lower-limb lymphedema, "case studies showing efficacy exist, but the quality of the evidence is low and should be implemented with caution (D2: low-quality level of evidence, or no recommendation rating). Internationally, exercise therapy's effectiveness for secondary upper-limb lymphedema after breast cancer has been demonstrated, but there are few studies on secondary lower-limb lymphedema [4] [6]. However, Monobe states that it is necessary to promote muscle strength maintenance exercise while monitoring the state of edema, as the muscle pumping action of moving muscles improves lymphatic drainage, and edema is aggravated when it is decreased [12]. Ogawa and Sato 
state that daily exercise such as walking is effective, and if walking is difficult, then bending and stretching of the knee and ankle joints, toe exercises, and knee kicking on the bed are also effective [13] [14].

As described above, once lymphedema develops it is intractable. Preventive self-care is important, but it is difficult to continue self-care especially in cases where the disease has not yet developed or is mild enough to not interfere. Therefore, we consider that a continuable self-care supporting program for patients with latent or mild secondary lower-limb lymphedema plays an important role.

The purpose of this study was to consider the feasibility of continuing a self-care supporting program for patients with early stages of secondary lower-limb lymphedema that incorporates "simple exercises to replace lymphatic drainage" based on lymphatic flow.

\section{Methods}

\subsection{Overview of the Structure of the Self-Care Supporting Program}

The program aims to make patients with early stages of lymphedema interested in understanding lymphedema and encourage them to continue caring for themselves. This program has two features. First, the information provided is minimized, and the content of self-care practiced is also minimized. Second, it incorporates "Simple exercises to replace lymphatic drainage" based on lymphatic flow. These simple exercises contain elements of "Lymphatic drainage" and "Exercise" that are considered difficult to continue in self-care. The program is summarized as follows (Figure 1).

A) Targeted patients of the program: Patients with early stages of secondary lower-limb lymphedema (ISL stage 0 to stage I).

B) Self-care contents: The information to be provided is related to "Understanding lymphedema," "Self-monitoring (Observation, Touching, Measurement)," "Self-care (Skin care, Simple exercises to replace lymphatic drainage, Compression, Other exercises)," and "Precautions in daily life." The leaflet that summarizes this information was printed on one A3 size sheet and folded in half (A4 size ${ }^{\star} 4$ pages).

1) Understanding lymphedema:

The mechanism of lymphedema and the classification of edema progression by ISL stage will be explained. Acknowledge that patients who have undergone surgery with lymphatic dissection can lead to edema, and the self-care for prevention in stage 0 to stage I for them is important.

2) Self-monitoring:

a) Observation: Visually check the color, dryness, and scars of the skin daily.

b) Touching: Check if there is any swelling status, fever, and hardness by touching the skin daily.

c) Measurement: Check bodyweight and five lower-limb circumferences (ankle, calf, knee, thigh, groin) once a week. 


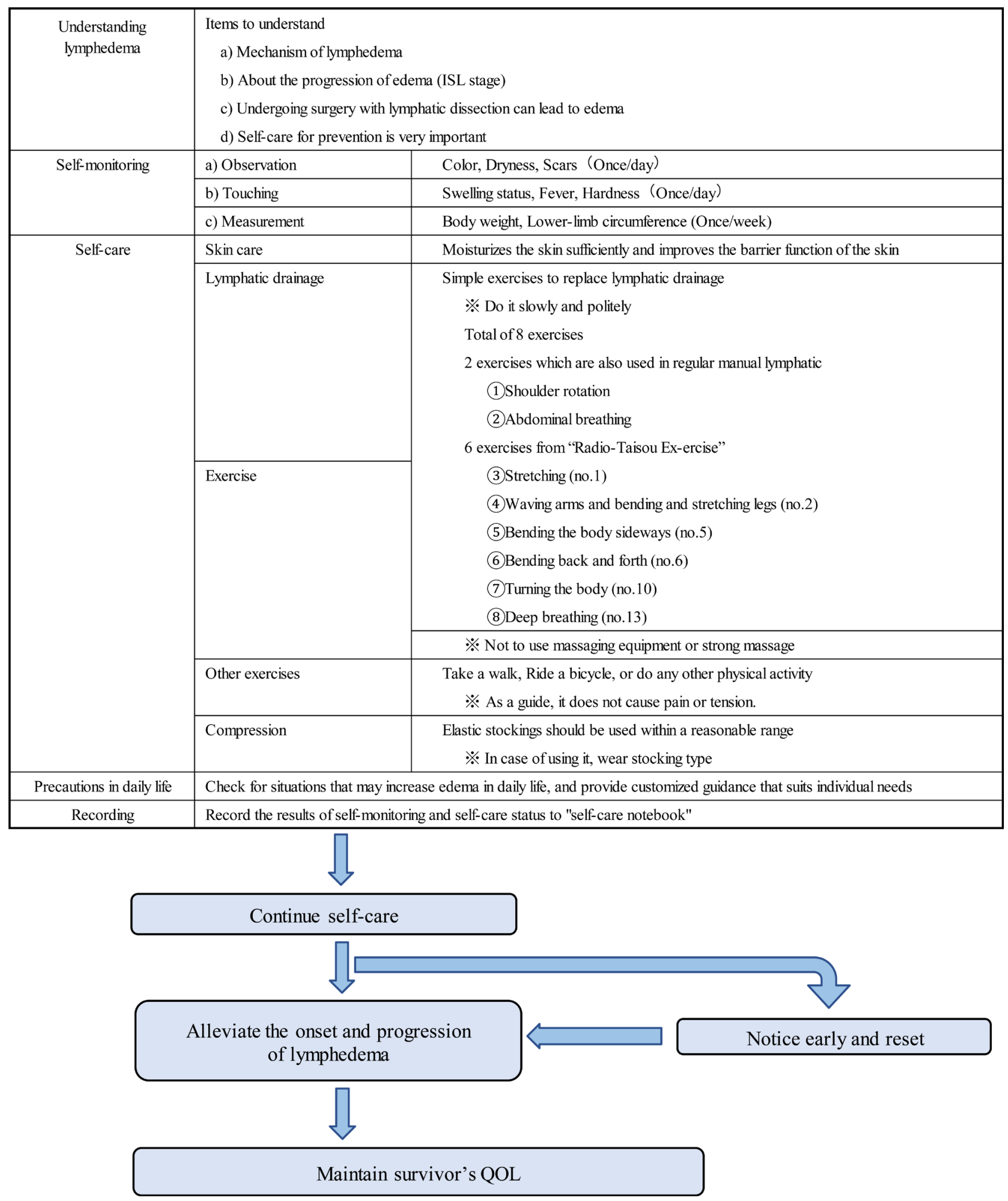

Figure 1. Structure of this self-care supporting program.

3) Self-care:

a) Skin care: It is important to moisturize the skin sufficiently to improve the barrier function of the skin. 
b) Simple exercises to replace lymphatic drainage: first perform "Shoulder rotation" and "Abdominal breathing," which are also used in regular manual lymphatic drainage, and then add six exercises from "Radio-Taisou Exercise [15]" for a total of eight exercises; "Stretching (no. 1)," "Waving arms and bending and stretching legs (no. 2)," "Bending the body sideways (no. 5)," "Bending back and forth (no. 6)," "Turning the body (no. 10)," and "Deep breathing (no. 13)". It takes about eight minutes. Explaining to them that it is especially important to do this before bedtime to reset the lymphatic system which is stagnant during sleep. Create a video and uploaded it to a video streaming site to be viewed when required. Careful not to use massaging equipment or strong massage, as it may damage the lymphatic vessels. Before this study, the effect of this "simple exercise to replace lymph drainage" was tested by comparison before and after exercise, confirming a reduction in lymphedema in patients up to stage II (Wilcoxon signed-rank test: $\mathrm{z}=-2.97, \mathrm{r}=0.70, \mathrm{p}=0.03$ ) [16].

c) Compression: Elastic stockings should be used within a reasonable range. In case of using it, wear a stocking type and make sure to let the lymph fluid flow to the upper body.

Be careful not to hurt the skin.

d) Other exercises: Take a walk, ride a bicycle, or do any other physical activity as long as it does not cause pain or tension.

4) Precautions in daily life: Check for situations that may increase edema in daily life, and provide customized guidance that suits individual needs.

C) Recording: Use the "Self-care notebook" to record and visualize the results of self-monitoring and self-care status. The notebook is A4 size and has one page for each week.

\subsection{Study Design}

This study is a descriptive study on the feasibility of continuing a self-care supporting program that was devised.

\subsection{Survey Period}

June 2020-December 2020.

\subsection{Study 1: Self-Care Continuity Status}

\section{A) Objective}

To assess the patient's self-care continuity status.

\section{B) Participants}

1) Inclusion criteria were patients: a) with gynecological cancer who had undergone surgery or radiation therapy with lymph node dissection, $b$ ) with early stages of secondary lower-limb lymphedema from stages 0 to I or II close to stage I, c) who rarely performed self-care, d) with ability to light exercise, and e) with approval of the attending physician to participate in the study.

2) Exclusion criteria were patients: a) with signs of inflammation such as cel- 
lulitis, b) with thrombosis or serious cardiovascular disease, c) with musculoskeletal disorders or disabilities, d) with brain and nerve system disorder or cognitive impairment, e) who were unable to communicate in Japanese, and f) who were minors.

C) Procedures

1) We explained to qualified patients in writing and verbally about their participation in the study and obtained signed consent.

2) A self-administered questionnaire was used to collect data on age, stages of lymphedema, medical diagnosis, surgical procedure, postoperative years, treatment other than surgery, lymphedema status, and self-care status.

3) We used leaflets to provide information on "understanding lymphedema," "self-monitoring," "self-care" and "precautions in daily life".

4) "Simple exercises to replace lymphatic drainage" was performed while reviewing the caution points.

5) Patients were required to continue self-care at home and record their status in the self-care notebook.

6) After a month or more, a self-administered questionnaire was used to collect data on the self-care continuity status and perceptions toward self-care.

7) The self-care notebooks were collected for one month (notebooks were returned later).

\section{D) Evaluation items and analysis}

For the "self-care continuity status," we analyzed the continuity status of "observation," "touching," "measurement," "skin care," "simple exercises to replace lymphatic drainage," "compression," "other exercises," and "recording" based on the contents of the self-administered questionnaire and self-care notebooks.

For the "perceptions toward self-care," we used a self-administered questionnaire in five stages to ask the respondents whether they think each item of self-care is "important to me," or "burden to me." We aggregated "I do agree on this so much" and "I agree" into "I think it is important to me" and "I think it is a burden to me," respectively. Similarly, we aggregated "I do not agree on this so much" and "I do not agree" into "I do not think it is important to me" and "I do not think it is a burden to me," respectively. The items of self-care are "observation," "skin care," "lymphatic drainage," "exercise" and "measurement".

\subsection{Study 2: Therapist's Opinions}

\section{A) Objective}

To listen to lymphedema therapists' opinions about the program and discuss the feasibility of continuing a self-care supporting program and points for improvement.

\section{B) Participants}

Certified lymphedema therapists with at least five years of experience.

C) Procedures

1) We explained to qualified therapists both in writing and verbally regarding their participation in the study and obtained signed consent. 
2) After explaining the purpose and contents of this program, we asked for their opinions about the program as a whole, items included in the program, simple exercises to replace lymphatic drainage, the leaflet, and the self-care notebook.

3) The interview was recorded after obtaining consent from the therapists.

4) Comments were extracted from the interview content regarding the feasibility of continuing a self-care supporting program and its points for improvement.

\subsection{Ethical Considerations}

We explained to the respondents both in writing and orally regarding the contents of the study that, they have the right to participate or exit from the study, they could withdraw their intention to participate even after cooperating in the study, and there would be no disadvantages to their treatment or care, as well as their personal information would be kept confidential. We confirmed our intentions by signing the consent form. This study was conducted under the approval of the Ethics Committee of Osaka Medical College (Approval number: KAN-148 [2894]).

\section{Results}

\subsection{Study 1: Self-Care Continuity Status}

1) Overview of the participants (Table 1)

The participants were 6 women. The median age (range) was 57.5 (41 to 68) years. The postoperative years were 8.5 ( 1 to 14 ) years. Stages of lymphedema were ISL Stage 0 in one patient, Stage I in one patient, and Stage II (close to Stage I) in four patients. The medical diagnosis was endometrial cancer in three patients, cervical cancer in one patient, ovarian cancer in one patient, and endometrial cancer plus ovarian cancer in one patient. All six patients had undergone surgery with lymphatic dissection.

2) Self-care continuation status after one month (Table 2)

We assessed the continuation of self-care based on the content of the self-administered questionnaire survey and self-care notebooks. "Observation," "Touching," "Skin care," and "Simple exercises to replace lymphatic drainage" were performed almost daily by all participants. "Measurement," the lower-limb circumferences were measured at least once a week by two participants, once a month by two, and zero times by the remaining two. Bodyweight was measured at least once a week by four participants, once a month by one, and zero times by the remaining one. As for "Compression," four of the patients used elastic stockings for compression every day. The two patients who did not do any compression had stage 0 and stage I edema. "Other exercises" were conducted every day by three participants, three to four times a week by one, and the remaining two did not other exercises. "Recording" was continuously kept by five participants, but the remaining one barely kept records. The edema level of the participant who did not continuously conduct "Measurement," "Other exercises" and "Recording" was stage I.

3) Participants' perceptions about self-care after one month (Table 3) 
Table 1. Overview of the participants $(\mathrm{N}=6)$.

\begin{tabular}{|c|c|c|}
\hline Item & Response & Number \\
\hline Age & Median: $57.5(41-68)$ & \\
\hline Postoperative Years & Median: $8.5(1-14)$ & \\
\hline \multirow[t]{3}{*}{ Stage of lymphedema } & ISL Stage 0 & 1 \\
\hline & ISL Stage I & 1 \\
\hline & ISL Stage II (Close to stage I) & 4 \\
\hline \multirow[t]{4}{*}{ Medical Diagnosis } & Endometrial cancer & 3 \\
\hline & Cervical cancer & 1 \\
\hline & Ovarian cancer & 1 \\
\hline & Endometrial and Ovarian cancer & 1 \\
\hline \multirow[t]{5}{*}{ Surgical Procedure } & Total hysterectomy & 3 \\
\hline & Extended hysterectomy & 1 \\
\hline & Bilateral salpingoophorectomy & 1 \\
\hline & $\begin{array}{l}\text { Total hysterectomy } \\
\text { and Bilateral salpingoophorectomy }\end{array}$ & 1 \\
\hline & Lymph node dissection & 6 \\
\hline \multirow[t]{3}{*}{ Treatment Other than Surgery } & Chemotherapy & 4 \\
\hline & Chemotherapy and Radiation therapy & 1 \\
\hline & None & 1 \\
\hline
\end{tabular}

Table 2. Self-care continuation status after one month $(\mathrm{N}=6)$.

\begin{tabular}{|c|c|c|c|c|c|c|c|c|c|}
\hline \multirow[b]{2}{*}{ Frequency } & \multirow[b]{2}{*}{ Observation } & \multirow[b]{2}{*}{ Touching } & \multicolumn{2}{|c|}{ Measurement } & \multirow[b]{2}{*}{$\begin{array}{l}\text { Skin } \\
\text { care }\end{array}$} & \multirow{2}{*}{$\begin{array}{c}\text { Simple } \\
\text { exercises } \\
\text { (drainage) }\end{array}$} & \multirow[b]{2}{*}{ Compression } & \multirow[b]{2}{*}{$\begin{array}{c}\text { Other } \\
\text { exercises }\end{array}$} & \multirow[b]{2}{*}{ Recording } \\
\hline & & & Body weight & $\begin{array}{c}\text { Lower-limb } \\
\text { Circumferences }\end{array}$ & & & & & \\
\hline Every day & 6 & 6 & 1 & 1 & 6 & 6 & 4 & 3 & 5 \\
\hline 3 - 4 times a week & & & 2 & & & & & 1 & \\
\hline Once a week & & & 1 & 1 & & & & 1 & \\
\hline Once a month & & & 1 & 2 & & & & & \\
\hline Rarely do & & & 1 & 2 & & & 2 & 1 & 1 \\
\hline
\end{tabular}

Table 3. Participants' perceptions about self-care after one month $(\mathrm{N}=6)$.

\begin{tabular}{cccccc}
\hline Item & Observation & Skin care & Lymphatic drainage & Exercise & Measurement \\
\hline $\begin{array}{c}\text { Important to me } \\
\text { YES }\end{array}$ & 6 & 6 & 6 & 6 & 6 \\
NO & 0 & 0 & 0 & 0 & 0 \\
Burden to me & & 2 & 2 & 4 & 5 \\
YES & 4 & 4 & 4 & 2 & 1 \\
NO & 4 & & & \\
\hline
\end{tabular}


A self-administered questionnaire survey was used to assess perceptions about self-care. All six participants saw "Observation," "Measurement," "Skin care," "Lymphatic drainage" and "Exercise" as being "important to me." Two participants answered that "Observation," "Skin care" and "Lymphatic drainage" were "burden to me"; four said "Exercise" was a "burden to me"; and five said "Measurement" was "burden to me." There was also an indication that "Recording" was troublesome.

\subsection{Study 2: Therapist's Opinions}

1) Summary of therapists

Six therapists participated in the study consisting of five nurses and one occupational therapist. All of them had at least five years of experience.

2) Therapist's opinions about the self-care supporting program (Table 4)

a) Regarding the program as a whole

There were no problems with the criteria.

b) Regarding the items included in the program

Since these items were already implemented in the study, they did not have any particular opinions.

c) Regarding the simple exercises to replace lymphatic drainage

The therapists did not have any negative comments regarding this. Positive comments included "simple," "not difficult," "easy to use due to the video streaming site," "effective for health maintenance and management," "easy to recommend to seniors," "can be used for upper-limb lymphedema patients," "can be used as an exercise guide" and "can be done with family members." Some constructive comments were: "safety pre-cautions are needed for seniors and patients with underlying diseases" and "guidance on when to do it is needed".

d) Regarding the leaflet

They thought that the leaflet was easy to read due to the appropriate size and number of words. Some have said that "it is difficult to visualize changes in skin color by only with the text" and that "photos are more impactful and easier to visualize."

e) Regarding the self-care notebook

One therapist mentioned that the "entry fields are narrow and difficult to write in." They suggested that the "self-care notebook" should be simplified so that seniors don't find it troublesome, and that smartphones can also be incorporated.

\section{Discussion}

\subsection{Feasibility of This Program Due to the Patient's Self-Care Continuity Status}

Among the items of the self-care support program, "Observation," "Touching" and "Skin care" were found to be easier to continue. The previous study suggested that "Lymphatic drainage" and "Exercise" were difficult to continue in 
Table 4. Therapist's opinions about the self-care supporting program.

\begin{tabular}{lll}
\hline & Therapist's opinions & On points for improvement \\
\hline $\begin{array}{l}\text { Items included in the } \\
\text { program }\end{array}$ & (There is no particular problem with program items.) & \\
\hline
\end{tabular}

\begin{tabular}{|c|c|c|}
\hline \multirow{7}{*}{$\begin{array}{l}\text { Simple exercises to replace } \\
\text { lymphatic drainage }\end{array}$} & $\begin{array}{l}\text { As "Radio-Taisou Exercise" is a whole-body exercise, and I feel it is } \\
\text { good not only for lymphedema but also for maintenance and } \\
\text { improvement of health. }\end{array}$ & \\
\hline & $\begin{array}{l}\text { I advise people who do yoga, etc., to keep on doing it, but it is } \\
\text { difficult for people lacking fitness habits, especially elderly persons, } \\
\text { to change habits. "Radio-Taisou Exercise" is well-known amongst } \\
\text { everyone, so it is easy to recommend it. }\end{array}$ & \\
\hline & $\begin{array}{l}\text { I recommend "Radio-Taisou Exercise," saying, "Please watch a TV or } \\
\text { a video distribution service, listen to their advice carefully, and do it } \\
\text { according to their instructions." I thought it is really good to do it } \\
\text { together while showing them points to remember. }\end{array}$ & $\begin{array}{l}\text { Because there are elderly persons, people with } \\
\text { underlying diseases, or people in bad shape, it } \\
\text { is necessary to be careful about safety. }\end{array}$ \\
\hline & $\begin{array}{l}\text { Even if people are given an explanation with a pamphlet, etc., it is } \\
\text { hard for them to visualize. Doing it together or by uploading a video } \\
\text { on streaming site, makes them understand it more easily. } \\
\text { Because it is supposed to be uploaded on a video streaming site, they } \\
\text { can do it whenever they want. }\end{array}$ & $\begin{array}{l}\text { I advise people who have difficulty continuing } \\
\text { it to "Please do it as if it were a medicine after } \\
\text { meals." It is also necessary to give them } \\
\text { instructions about when it should be done in } \\
\text { accordance with a person's lifestyle. }\end{array}$ \\
\hline & $\begin{array}{l}\text { Many people feel overloaded when they do "Radio-Taisou Exercise" } \\
\text { carefully and completely. If it is too burdensome, it becomes difficult } \\
\text { to continue. So, it is interesting to present the minimum necessary } \\
\text { motions from the perspective of the flow of lymph. }\end{array}$ & \\
\hline & $\begin{array}{l}\text { As Upper-limbs exercises are included, I think it can be used for } \\
\text { patients with breast cancer. }\end{array}$ & \\
\hline & $\begin{array}{l}\text { Because it is possible to explain it to family members too, I guess they } \\
\text { too can participate. }\end{array}$ & \\
\hline \multirow{3}{*}{ Leaflet } & The leaflet has an appropriate volume and is easy-to-read. & \\
\hline & $\begin{array}{l}\text { As there is both a leaflet and a video, it is possible care for outpatients } \\
\text { using them together. }\end{array}$ & $\begin{array}{l}\text { I think it may be difficult to visualize skin } \\
\text { color changes, and so on, only through words. }\end{array}$ \\
\hline & $\begin{array}{l}\text { People with lower extremity lymphedema tend to think that they } \\
\text { must not exercise. I think they may feel safe because there is a guide } \\
\text { (standard) on how much exercise can be allowed. }\end{array}$ & $\begin{array}{l}\text { If photos are included, it will be better, and } \\
\text { may become easier to visualize. }\end{array}$ \\
\hline \multirow{4}{*}{ Self-care notebook } & & $\begin{array}{l}\text { When patients are explained/keeping a record, } \\
\text { they lose interest in it. }\end{array}$ \\
\hline & & $\begin{array}{l}\text { Patients interested in keeping a record are in } \\
\text { early stage and can continue self-care easily. }\end{array}$ \\
\hline & & $\begin{array}{l}\text { Because the entry columns are too small, } \\
\text { people may have difficulty filling them out. }\end{array}$ \\
\hline & & $\begin{array}{l}\text { As many patients have smartphones, it is } \\
\text { better to make use of them. }\end{array}$ \\
\hline
\end{tabular}

self-care, yet they were successfully continued as "Simple exercises to replace lymphatic drainage." We observed many cases where the self-manual lymphatic drainage was stopped less than a month but found the "Simple exercises to replace lymphatic drainage" might be continuable. Regarding "Compression," the stage II (close to stage I) participants wore them daily. As for "Other exercises," 
three out of six participants performed daily, and two performed three to four times a week. The suggested exercises, such as walking and riding a bicycle, were among the participants' daily activities and readily continued by adding the extra distance or hours.

While all items in the self-care supporting program were important, "Measurement" and "Other exercises" were seen as burdens. This dovetail with the conclusion of the previous study, suggesting the difficulty of continuing the self-care. The participants were relatively successful at continuing "Observation," "Touching" and "Skin care" because they could see or touch the symptom when they bathed, thus performed these items as part of their daily activities. However, "Measurement" and "Other exercises" were either non-daily or physically taxing activities, hence required some special motivators to continue. It is deemed essential to determine the most appropriate timing in their everyday lives to perform these activities on a case-by-case basis in collaboration with the participants.

The keystone of this program was the "Simple exercises to replace lymphatic drain-age," which contains elements of "Lymphatic drainage" and "Exercise." "Simple exercises to replace lymphatic drainage" consisted of eight different exercises, "shoulder rotation" and "abdominal breathing" which are regularly included in manual lymphatic drainage practice, plus six movements from "Radio-Taisou Exercise" that based on lymphatic flow. The "Radio-Taisou Exercise," the exercise carried out under the music, is a series of aerobic exercises for upper and lower limbs and body trunk. The key elements were skin extension/loosening required to draw lymphatic fluid in lymph capillaries and adequate stress required to maintain the muscle pumping to facilitate lymphatic fluid transport. For an effective implementation, a slower pace (about 50 Beats Per Minute (BPM)) than usual (about $70 \mathrm{BPM}$ ) was set, and the participants performed each movement with careful attention to the key elements. The duration was eight minutes and it was considered that this exercise could be continued with a busy life and was less time-consuming. Everyone in Japan, whether children or the elderly, are familiar with this exercise.

For patients with latent or mild lymphedema, the prevention of lymphedema is believed to lead to maintaining their QOL. However, continuing self-care can be stressful for them. It should be reasonable to assume that self-care continues to stress the participants that selected "I have to do it" to some extent.

The "Recording" feedback suggested it required too many details to submit easily and did not encourage the participants to continue self-care daily. It indicated the need to limit the number of self-care activities to a carefully selected bare minimum. Maintaining the records can help the patients realize the effect of self-care and maintain self-efficacy through visualization. Self-care notebooks can be improved to be more straightforward and motivating. Today, elderly patients can use smartphones and it may be useful to develop a smartphone app that is easy to operate and visually realize the effect. 


\subsection{Feasibility of This Program Due to Therapist's Opinions}

The evaluation of this program by therapists was generally positive. The therapists' approval was considered quick and easy, as the program had the same structure as the regular program but including situational exercises like "Lymphatic drainage" and "Exercise". The URL and the QR-code provision for the exercise video were also beneficial when giving the lymphedema prevention guidance. Regarding "recording," it was highlighted that the fields in the self-care notes were too detailed. We therefore need to improve the items and styles described in our self-care notes. We also want to consider developing and utilizing smartphone apps.

\subsection{Research Limits and Future Challenges}

This study is a descriptive study of the sustainability of self-care support programs, and it is difficult to generalize the results because the participants were limited to 6 patients and 6 therapists. However, we are able to update to a more straightforward and effective program based on the improvements found in this study. Furthermore, future research is required to understand the long-term effects of this program on the prevention of lymphedema.

\section{Conclusions}

We considered the feasibility of continuing a self-care supporting program for patients with early stages of secondary lower-limb lymphedema and obtained the following results.

1) The patients understood the importance of all items in the program and were able to continuously practice "Observation," "Touching" and "Skin care". "Lymph drainage" and "Exercise" were continued through "Simple exercise instead of lymph drainage".

2) "Other exercises" was a burden on the patient but could be continued by adding distance and time to daily activities such as walking and cycling.

3) "Measurement" and "Recording" were a burden for the patient and difficult to carry out continuously.

4) This program was generally approved by the therapist. However, the need for improvement in the content and format of "Recording" was required.

Therefore, the feasibility of continuing this program has been suggested. It also revealed improvements that could further update the program.

\section{Acknowledgements}

We would like to thank the patients, therapists and the staff of the medical institution who participated and cooperated in the study. Sincere, thanks to S, Miyazaki for assisting in this research study. We would also like to thank Professor Michishige, Professor Dote, and Associate Professor Kubota for their expertise and advice. 


\section{Authors Contributions}

CAr conducted the entire study under the supervision of CAk and prepared the manuscript. CAk and ST were involved in the acquisition, analysis, interpretation of data. All authors critically revised the report, commented on drafts of the manuscript, and approved the final report.

\section{Conflicts of Interest}

The authors declare no conflicts of interest regarding the publication of this paper.

\section{References}

[1] Japan "Sociology of Cancer" Study Group and Yamaguchi, K. (2013) Voice of 4,054 People Facing Cancer. Survey Report on Cancer Survivors Worries and Burdens. https://www.scchr.jp/book/houkokusho/2013taikenkoe.html

[2] National Cancer Center Japan (2020) Cancer Registration and Statistics. https://ganjoho.jp/reg stat/statistics/stat/summary.html

[3] International Society of Lymphology Executive Committee (1995) The Diagnosis and Treatment of Peripheral Lymphedema. Lymphology, 28, 113-117.

[4] International Society of Lymphology Executive Committee (2020) The Diagnosis and Treatment of Peripheral Lymphedema: 2020 Consensus Document of the International Society of Lymphology. Lymphology, 53, 3-19.

[5] Joachim, E. and Steve, N. (2017) Lymphedema Management: The Comprehensive Guide for Practitioners. 4th Edition, Thieme, New York.

[6] International Lymphoedema Framework and Christine, M. (2006) Best Practices for the Management of Lymphoedema. Hargrave Road, London.

https://www.lympho.org/portfolio/best-practice-for-the-management-of-lymphoed $\underline{\text { ema }}$

[7] Ministry of Health, Labor and Welfare (2008) Lymphedema Guidance and Management Fee, Calculation Method of Medical Fees. No. 59, B001-7.

https://www.mhlw.go.jp/web/t doc?dataId=84aa9729\&dataType $=0$ \&pageNo $=4$

[8] Akazawa, C., Fukuda, R., Arakawa, C. and Yamamoto, M. (2011) Questionnaire Survey of Patients with Secondary Lower-Limb Lymphedema (2nd Report)-Relationship between Self-Care Continuation and Self-Efficacy. Journal of Japan Academy of Nursing Science, 34, 469.

[9] Akazawa, C., Fukuda, R., Honda, I., Arakawa, C. and Yamamoto, M. (2012) Consideration of Body Water Fluctuation by Manual Lymphatic Drainage Treatment Only on the Upper Body by BIA Composition Analyzer. Journal of Japan Academy of Nursing Science, 35, 309.

[10] Akazawa, C., Fukuda, R. and Arakawa, C. (2015) Examination of Evaluation Methods to Support the Continuation of Self-Care in Patients with Lower-Limb Lymphedema. Journal of Japan Academy of Nursing Science, 38, 312.

[11] The Japanese Lymphedema Society (2018) Lymphedema Clinical Practice Guideline. Kanehara, Tokyo.

[12] Monobe, C. (2016) Practice and Daily Life Guidance for Lymphedema Care. Journal of Nursing Practice, 62, 39-43.

[13] Ogawa, Y. (2013) Conservative Treatment of Lymphedema Based on Evidence. 
Journal of Japanese College of Angiology, 24, 447-456.

https://doi.org/10.7134/phlebol.414

[14] Sato, K. and Ogawa, Y. (2010) Treatment and Care of Lymphedema. 2nd Edition, Igaku-syoin, Tokyo.

[15] Japan Post Insurance. Illustrated Radio-Taisou Exercise 1st, Standing Version. https://www.jp-life.japanpost.jp/radio/instruction/radio first.html

[16] Arakawa, C., Akazawa, C. and Teraguchi, S. (2020) Examination of the Effect of Exercise Replacing Manual Lymph Drainage for Patients with Secondary Lower-Limb Lymphedema. Journal of Osaka Medical College, 79, 71-79. 